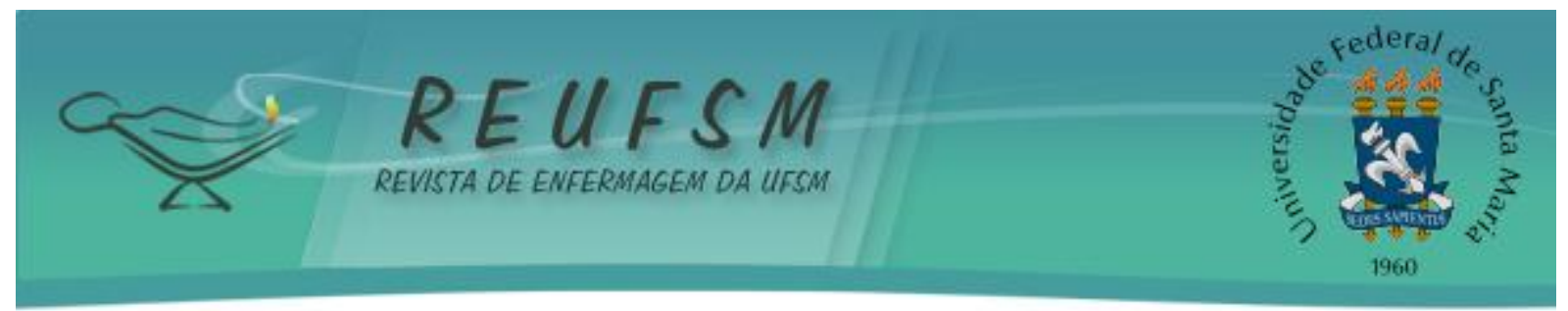

ARTIGO ORIGINAL

\title{
INCIDÊNCIA DE ÚLCERA POR PRESSÃO COMO INDICADOR DE QUALIDADE NA ASSISTÊNCIA DE ENFERMAGEM
}

\author{
INCIDENCE OF PRESSURE ULCERS AS HEALTHCARE QUALITY INDICATORS IN NURSING \\ CARE
} INCIDENCIA DE ÚLCERAS POR PRESIÓN COMO INDICADOR DE CALIDAD EN EL CUIDADO DE
ENFERMERÍA

Mara Rosane Vargas e Silva ${ }^{1}$ Nidea Rita Michels Dick ${ }^{2}$ Angela Conte Martini ${ }^{3}$

RESUMO: Objetivo: analisar a incidência de úlcera por pressão como indicador de qualidade assistencial no Hospital Coronel Frota/Porto Alegre. Método: estudo quantitativo que se valeu dos princípios da exploração e descrição, utilizando-se a análise estatística das notificações do evento adverso úlcera por pressão. Resultados: foram identificados baixos índices de úlceras por pressão. Os pacientes acometidos apresentavam diversos fatores de risco incluindo alterações nutricionais, de mobilidade e de nível de consciência. Destacou-se como cuidados de enfermagem na prevenção de úlceras por pressão o uso de colchão piramidal e a mudança de decúbito. Considerações finais: apesar de se constatar a existência do cuidado de enfermagem preventivo, mesmo assim houve o desenvolvimento de úlcera por pressão. Evidenciou-se a necessidade de ampliar as ações de prevenção de úlceras por pressão bem como, avaliar a acurácia das notificações dos eventos adversos. Descritores: Úlcera por pressão; Qualidade da assistência à saúde; Cuidados de enfermagem.

ABSTRACT: Objective: to analyze the incidence of pressure ulcers as a healthcare quality indicator at Coronel Frota Hospital in Porto Alegre. Method: a quantitative study based on the principles of exploration and description, which used statistical analysis of adverse event reports of pressure ulcers. Results: low pressure ulcer rates were identified. The affected patients had several risk factors including changes in nutrition, mobility and level of consciousness. The use of pyramidal mattress and the position change were highly indicated as nursing care in the prevention of pressure ulcers. Concluding Remarks: pressure ulcers developed despite the existence of preventive nursing care. The need to expand pressure ulcer prevention efforts and to evaluate the accuracy of adverse event reporting was identified.

Descriptors: Pressure ulcers; Quality of health care; Nursing care.

RESUMEN: Objetivo: analizar la incidencia de úlcera por presión como indicador de calidad asistencial en el Hospital Coronel Frota en la ciudad de Porto Alegre. Método: estudio cuantitativo que se basó en los principios de exploración y descripción y utilizó el análisis estadístico de los informes de eventos adversos de úlcera por presión. Resultados: fueron identificadas bajas tasas de úlcera por presión. Los pacientes afectados tenían varios factores de riesgo, incluyendo cambios en la alimentación, en la movilidad y en el nivel de

\footnotetext{
${ }^{1}$ Enfermeira. Especialista em Administração Hospitalar, Auditoria em Saúde, Segurança Pública, Oficial do Quadro de Saúde da Brigada Militar. Porto Alegre, RS, Brasil. E-mail: mara@brigadamilitar.rs.gov.br

${ }^{2}$ Enfermeira. Especialista em Administração Hospitalar, Enfermagem do Trabalho, Enfermagem Pediátrica, Segurança Pública, Oficial do Quadro de Saúde da Brigada Militar. Porto Alegre, RS, Brasil. E-mail: nidea@brigadamilitar.rs.gov.br

${ }^{3}$ Enfermeira. Mestre em Enfermagem. Docente do Centro Universitário Lasalle/Unilasalle - Canoas/RS, Brasil. Email: amartini@unilasalle.edu.br
} 


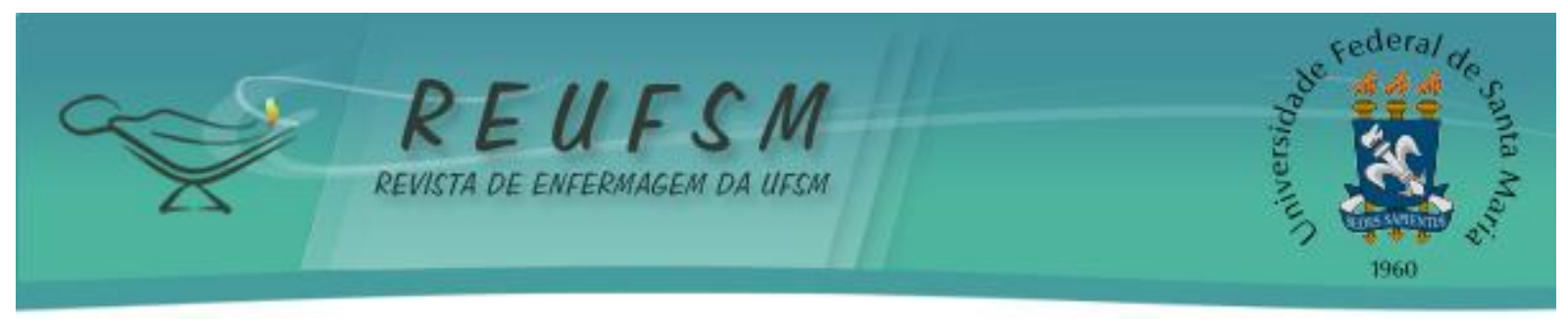

conciencia. Se destacaron como cuidados de enfermería en la prevención de úlceras por presión el uso del colchón piramidal y el cambio de posición. Consideraciones finales: la úlcera por presión se desarrolló a pesar de haber cuidados de enfermería preventiva. Se demostró la necesidad de ampliar los esfuerzos de prevención de úlceras por presión y también de evaluar la exactitud de los informes de eventos adversos.

Descriptores: Úlceras por presión; Calidad de la atención de salud; Cuidados de enfermería.

\section{INTRODUÇÃO}

O foco da enfermagem enquanto ideal teórico está no cuidado ao ser humano, na melhoria das condições de saúde, na prevenção de doenças e, até mesmo, em uma morte digna. Por existirem discrepâncias entre o ser e o fazer da enfermagem, a assistência à saúde pode gerar agravos que, primeiramente, se apresentam como riscos, mas que podem evoluir gerando eventos adversos que podem levar a seqüelas ou morte. Esta realidade faz com que profissionais da saúde mobilizem ações na tentativa de aproximação entre teoria e prática.

Entre os agravos nas condições de saúde de pacientes hospitalizados está o desenvolvimento de úlceras por pressão (UP). As UP são lesões causadas por isquemia secundária à compressão e que acontecem por período longo, quando diminui a irrigação sanguínea para a rede capilar prejudicando o fluxo sanguíneo para os tecidos circundantes e privando os tecidos de oxigênio e nutrientes. Isso gera isquemia local, hipóxia, edema, inflamação e morte celular, também denominada úlcera de decúbito ou escara. É comum em áreas onde há comprometimento da circulação, que acontece em função da compressão. Este comprometimento pode ser superficial, causado por irritação cutânea local, ou profundo, originado de tecidos subjacentes. ${ }^{1}$

Os riscos para a lesão de pele envolvem fatores extrínsecos/externos e intrínsecos/internos. São conhecidos como fatores externos: a pressão, identificada como o mais importante; as forças de cisalhamento, que podem deformar e destruir o tecido e acontecem quando o paciente desliza da cama; e a fricção, que ocorre quando duas superfícies são esfregadas uma na outra, sendo a causa mais comum arrastar o paciente na cama. Já os fatores internos descrevem o estado geral no qual as pessoas doentes são mais vulneráveis: a idade, já que o envelhecimento torna a pele mais fina e menos elástica; a mobilidade reduzida, que é um dos principais fatores que influenciam em outros riscos, podendo estar associada a um déficit neurológico e a diversas outras patologias; entre outros fatores. ${ }^{2}$

A enfermagem desempenha um trabalho primordial para a prevenção das UP atuando no controle dos fatores de risco (pressão, cisalhamento, fricção, umidade, nutrição, capacidade de movimentação). Por esse motivo, a utilização de índices de úlcera de pressão tem sido associada a um cuidado de enfermagem de qualidade. Embora se reconheça o papel da enfermagem, considera-se que toda a equipe interdisciplinar tem seu papel na prevenção e no tratamento de UP. Além disso, existem ainda fatores intrínsecos de difícil manejo terapêutico que podem levar à UP e que envolvem diretamente todos os profissionais da saúde.

Ainda que se reconheça a complexidade da prevenção de UP, considera-se como meta diminuir pelo máximo a quantidade e a gravidade das úlceras de pressão. Desta forma, mesmo conhecendo todos os fatores que envolvem as UP, considera-se que altos índices de úlceras estão relacionados a uma assistência de enfermagem precária, enquanto que baixos índices de UP representam que a assistência à saúde está sendo superior aos fatores intrínsecos e extrínsecos passíveis de prevenção que são responsáveis pelas úlceras por pressão.

Garantir cuidados com a pele, nutrição e mobilidade adequados (para aliviar a pressão e promover a circulação) ajuda a prevenir a ocorrência de UP e, às vezes, quando estão no estágio I, possibilita revertê-las, além de controlar as mais profundas. ${ }^{1}$ 


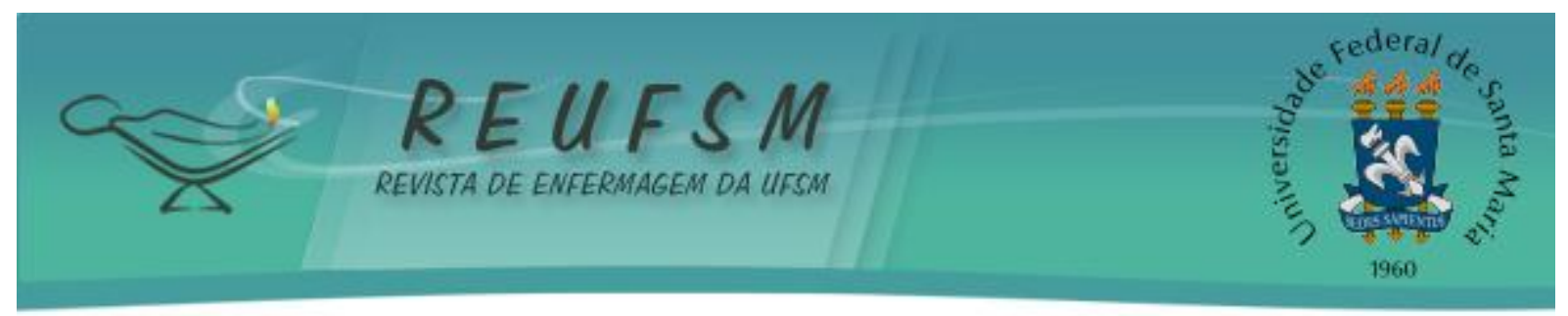

Medir qualidade e quantidade em programas e serviços de saúde é imprescindível para o planejamento, organização, coordenação/direção e avaliação/controle das atividades desenvolvidas. Os resultados, os processos e a estrutura necessária ou utilizada, bem como as influências e repercussões promovidas no meio ambiente, são alvos dessa medição. ${ }^{3}$

Neste sentido, objetivou-se analisar a incidência de úlcera por pressão como indicador da qualidade assistencial no Hospital Coronel Frota/Porto Alegre no ano de 2010.

\section{MÉTODO}

Trata-se de um estudo com abordagem quantitativa que tem por finalidade os princípios da exploração e descrição. A descrição quantitativa envolve a predominância, a incidência, o tamanho e os atributos mensuráveis de um fenômeno. A pesquisa exploratória investiga a sua natureza complexa e os outros fatores aos quais ele está relacionado. ${ }^{4}$

O campo de estudo foi o Hospital Público Militar Coronel Frota, situado na cidade de Porto Alegre, vinculado à Brigada Militar. Caracteriza-se por ser um hospital geral que atua na área de clínica e nas diversas especialidades de cirurgia. Possui 91 leitos subdivididos em 6 unidades. 0 grupo de enfermagem da instituição é composto por 23 enfermeiros e 124 técnicos de enfermagem, entre servidores militares e terceirizados.

No ano de 2009, o Hospital Coronel Frota iniciou o trabalho de construção, implantação e avaliação da qualidade assistencial. Partiu-se da definição de indicadores de qualidade assistencial, metodologia de coleta e análise dos dados.

Entre outubro a dezembro de 2009, iniciou-se um programa piloto de notificações de eventos adversos após o desenvolvimento de ações educativas.

No ano de 2010, consolidou-se o programa de indicadores de qualidade assistencial na enfermagem paralelamente e sistematicamente a um programa de educação em serviço e ao acompanhamento e controle de notificações por meio de constantes visitas e orientações nos setores. Entre os indicadores de qualidade, está o evento sentinela úlcera por pressão.

0 indicador assistencial - incidência úlcera por pressão - é obtido pela notificação utilizando um formulário próprio. Este formulário é composto por dados de identificação do paciente, incluindo sexo, idade, diagnóstico de patologias agudas e crônicas; condições do paciente, incluindo alterações de mobilidade; hidratação e alimentação; cuidados de enfermagem, incluindo mudança de decúbito e uso de colchão piramidal; características das lesões; descrição do evento adverso e observações.

Foram considerados, para fins de registro, todos os eventos referentes ao indicador pré-estabelecido. Para coleta dos dados, utilizou-se a análise documental, que utiliza a busca de informações na linguagem escrita. Trata-se de observação e análise voltadas às manifestações que foram registradas e às ideias elaboradas a partir delas. ${ }^{5}$

Os dados foram coletados no mês de abril de 2011, quando analisou-se o total de notificações realizadas de janeiro a dezembro de 2010 referentes a todas as unidades hospitalares, independentemente do risco de desenvolvimento de úlcera por pressão. A análise documental permitiu a identificação das notificações referente ao indicador de qualidade - úlcera por pressão - existentes na instituição.

A análise estatística ocorreu calculando-se percentuais e mediana. Os dados foram dispostos e analisados por meio de estatística descritiva, com utilização de médias, porcentagens e distribuição de frequência. "A distribuição de frequências é uma organização sistemática de valores numéricos do mais baixo ao mais alto, juntamente com a contagem (ou a porcentagem) do número de vezes que cada valor foi obtido". 4:213

Na realização desta pesquisa, manifestado o interesse no campo de estudo, foi solicitado o consentimento da instituição por meio de documento oficial próprio. Após, o projeto de pesquisa foi encaminhado para apreciação pelo Comitê de Ética e Pesquisa do Município de Porto 


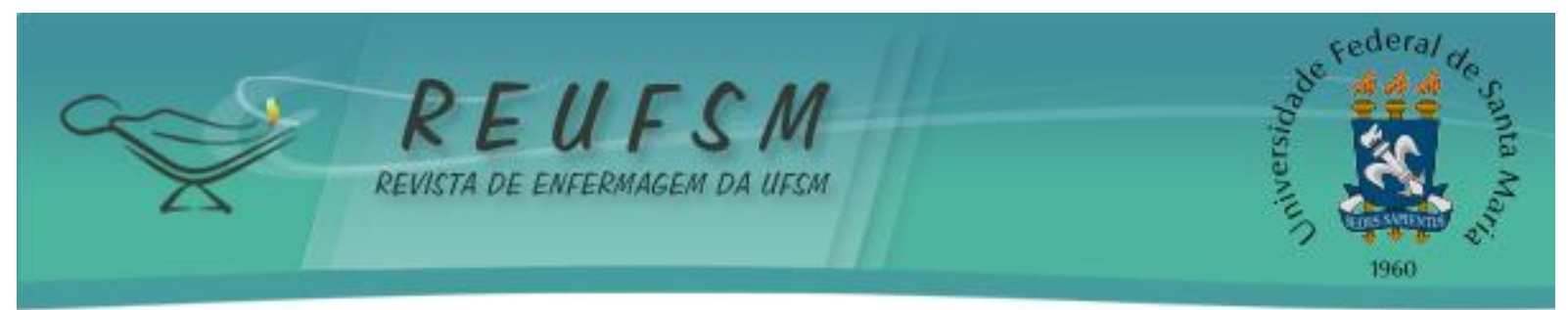

Alegre, sendo aprovado sob o número 001053402.10.3. Além disso, foi assinado um Termo de Compromisso de Utilização e Divulgação de Dados garantindo a utilização de informações codificadas para a elaboração da pesquisa a fim de preservar a confidencialidade e a privacidade dos sujeitos envolvidos indiretamente, sejam eles referentes a prontuários de pacientes ou profissionais que realizaram a notificação. Para a preservação dos aspectos éticos, foram respeitados os princípios referentes a pesquisas com seres humanos, descritos na Resolução 196/96 e suas complementares, do Conselho Nacional de Ética em Pesquisa. ${ }^{6}$

\section{RESULTADOS}

No Hospital Coronel Frota/Porto Alegre, as UP desenvolveram-se em 17 pacientes, perfazendo um total de 22 lesões em 21.227 pacientes ano (aproximadamente 1/1000). Estes casos foram distribuídos ao longo dos meses do ano de forma semelhante, não havendo picos ou períodos sem que acontecessem novos casos. Também foi observado que não houve alterações geográficas significantes em relação ao desenvolvimento de úlceras por pressão, nem mesmo em Unidade de Terapia Intensiva (UTI).

As 22 UP que acometeram os 17 pacientes apresentavam-se em diferentes regiões corporais. Verificou-se que $41 \%$ das lesões apareceram na região sacral, $32 \%$ no trocânter, $18 \%$ no calcâneo, $5 \%$ na escápula e $4 \%$ no maléolo.

Constatou-se que $35 \%$ das lesões foram classificadas como pequenas (até $5 \mathrm{~cm}$ ), $18 \%$ médias e $18 \%$ grandes. Nas demais $29 \%$, não foi informado o tamanho da lesão, evidenciando a necessidade de intensificar as atividades de educação e preenchimento das notificações de eventos adversos - úlcera por pressão. Destas lesões, $41 \%$ eram ulceradas, $29 \%$ hiperemiadas, $24 \%$ necrosadas e $6 \%$ com lesões bolhosas.

Um dos fatores que comprometem a pele é o grau de dependência, que normalmente favorece a fricção, cisalhamento, pressão, alteração da umidade da pele pelo uso de fraldas, entre outros fatores de risco. 0 grau de dependência foi avaliado por ser um fator de risco para o desenvolvimento de úlceras de pressão. Constatou-se que $72 \%$ foram considerados pacientes dependentes, $7 \%$ deambulavam com auxilio, $7 \%$ apenas sentavam com auxílio e $14 \%$ dos pacientes não eram dependentes ou não foi informado na notificação.

Avaliando o estado de hidratação e nutrição, 26\% apresentavam-se desidratados e $17 \%$ estavam desnutridos. Do total de pacientes, $64 \%$ alimentavam-se por via oral, porém somente $33 \%$ possuia uma boa aceitação e $67 \%$ apresentavam pouca aceitação da alimentação. Dos pacientes acima, 36\% recebiam alimentação por sonda nasoentérica.

Do total de pacientes que desenvolveram UP, 79\% encontravam-se em leitos com colchão piramidal. Além do uso de colção piramidal, é prática da enfermagem manter mudança de decúbito. Dentre os dados levantados observou-se que $72 \%$ dos pacientes se beneficiavam da mudança de decúbito, $7 \%$ não se beneficiavam e $21 \%$ não foi informado. Comparando estes dados com o grau de dependência dos pacientes que desenvolveram UP (79\%), percebe-se que a mudança de decúbito ocorreu conforme necessidade dos pacientes.

\section{DISCUSSÃO}

Foi identificada a baixa incidência de úlceras por pressão, inclusive em Unidades de Terapia Intensiva. Em UTI, diversos estudos ${ }^{7-9}$ apontam para altos índices de úlceras $(13,4 \%, 21 \%, 33 \%)$, fato este que não ocorreu neste estudo. Este indicativo leva a necessidade de ampliar o acompanhamento do processo de trabalho para identificação de possíveis falhas no processo de identificação e notificação do evento adverso e consequente subnotificação. 


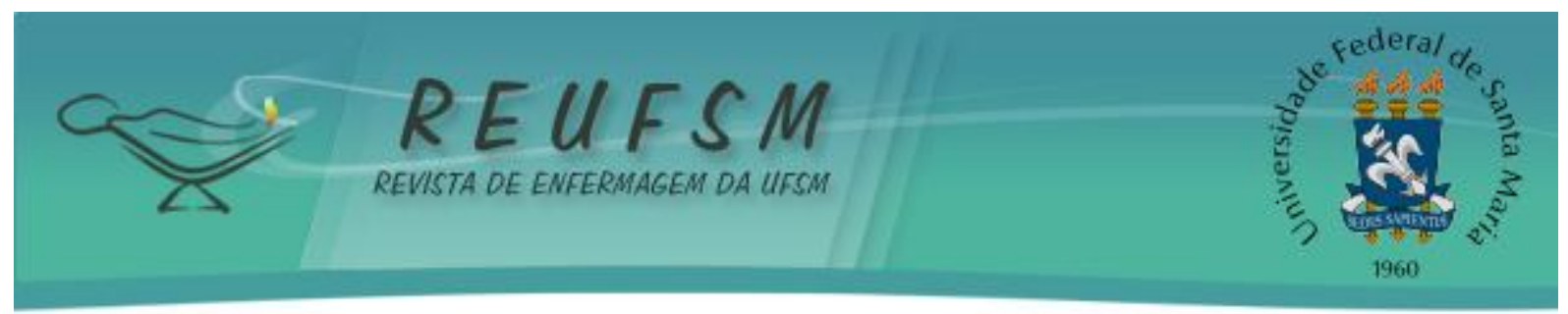

As UP se apresentaram em diversas regiões corporais e, conforme a convergência dos dados com a literatura, os locais mais comuns para formação de UP e que requerem maior cuidados são as regiões dos calcanhares, trocânteres, sacro, occípio dentre outros, sendo causadas por uma combinação de fatores intrínsecos e extrínsecos. ${ }^{10}$

Diversos são os fatores que levam ao desenvolvimento de úlceras por pressão. Entre eles estão as alterações nutricionais, a hidratação, o grau de dependência e a idade. Relacionados à ocorrência de UP estão os fatores de risco, sendo que estes foram encontrados em todos os pacientes, principalmente os relacionados a alterações nutricionais, de mobilidade e de nível de consciência.

Dentre os fatores extrínsecos estão o cisalhamento, a pressão e a fricção individualmente ou combinação. Já entre os fatores intrínsecos estão o estado nutricional do paciente, a idade, a mobilidade, a incontinência fecal do paciente, a umidade e a temperatura. Estes fatores interferem na capacidade da resistência tissular e nas interferências dos agentes causais externos relacionados a úlceras de pressão. ${ }^{10}$

A idade avançada produz modificações intensas no organismo humano, tornando-o mais propenso a doenças e lesões que podem tornar-se infecciosas e produzir sequelas e internações longas. ${ }^{11}$ Alterações circulatórias resultando da pressão, estado mental, umidade, atrito e forças de cisalhamento agravam a função tegumentar. ${ }^{12}$ Diabetes Mellitus, transtornos gastrointestinais e cardiopulmonares podem impedir o fluxo de nutrientes para os tecidos causando ulcerações. ${ }^{13}$ A nutrição desequilibrada é um fator de risco para o desenvolvimento de úlceras de pressão, como ficou evidenciado neste estudo. Além de aumentar o risco, comprometem a melhora e a cura das úlceras.

Sabe-se que a melhora e cura da ferida requer a ingestão adequada de nutrientes. Para pacientes que se encontram subnutridos ou desnutridos, as UP persistem e são mais graves, dificultando a cura da lesão. ${ }^{14}$

0 estado de saúde dos pacientes pode favorecer o desenvolvimento de UP. Entretanto, diversos são os fatores envolvidos que vão além das condições dos pacientes. Dessa forma, verifica-se que, para prevenir o problema, torna-se necessário utilizar abordagens que lidem com todo o sistema integrando o paciente e o ambiente institucional adequado. ${ }^{15}$ Neste estudo, se destacaram o uso de colchão piramidal e mudança de decúbito como cuidados na prevenção.

As recomendações de caráter preventivo instituídas por enfermeiros em sua prática diária visam evitar o surgimento de úlceras por pressão. As intervenções de enfermagem relacionadas com a prevenção devem abordar aspectos como: cuidados com a integridade da pele, uso de emolientes para hidratação, utilização de dispositivos para incontinência urinária e reeducação vesical, posicionamento no leito, observação diária da pele do pênis quando utilizado dispositivo para incontinência urinária, cuidados higiênicos e alimentação rica em vitaminas e proteínas aos primeiros sinais de UP, de acordo com a idade e condição clínica de cada cliente. ${ }^{11}$

Sabe-se que a prevenção de UP deve ser prioridade das instituições de saúde. Estas medidas acontecem quando se equipam as unidades hospitalares com material de alívio de zonas de pressão e monitoriza-se o grau de risco, incidência e prevalência. Esses devem ser os primeiros passos para a implementação de protocolos de prevenção e para a sensibilização das equipes para a problemática das UP. ${ }^{8}$

0 tratamento das UP deve ser implementado quando as medidas preventivas não forem suficientes. Os tratamentos incluem: o nível sistêmico, com objetivo da melhoria do estado nutricional e redução da infecção; o conservador, realizado no início do aparecimento das lesões; e o tratamento local, que incluía a limpeza cirúrgica, curativos e coberturas. ${ }^{15}$ 


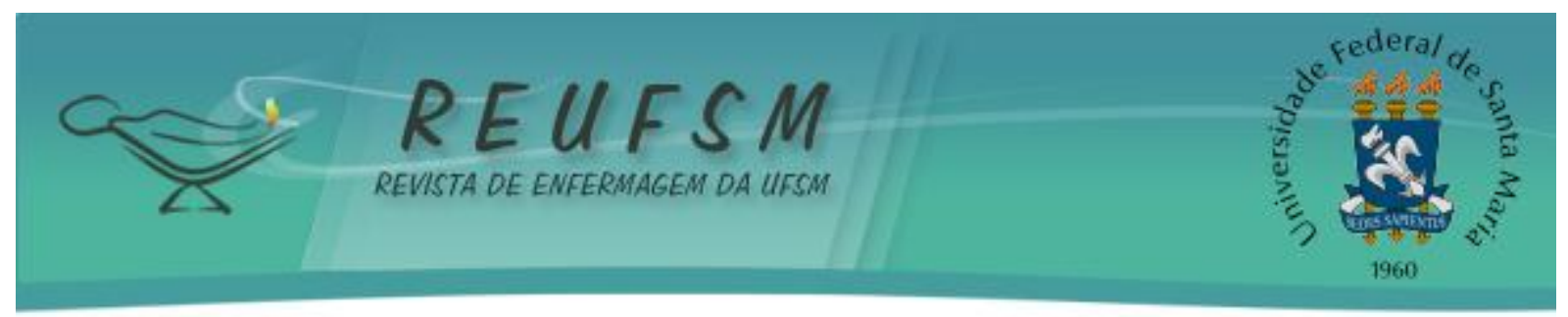

A UP é de difícil tratamento, em geral prolongado e oneroso, o que corrobora para a premissa da prevenção. Portanto, diante de pessoas que apresentem riscos para o desenvolvimento de úlceras por pressão, a equipe multiprofissional tem a responsabilidade de implementar medidas preventivas com o intuito de diminuir o impacto desse agravo. ${ }^{16}$

Pequenas mudanças na posição envolvem mover o paciente, mantendo a posição deitada, mas mudando o ângulo do lado em que ele está deitado ou alterando a posição do membro inferior na posição supina. Desta forma, ambas são estratégias úteis para auxiliar na perfusão dos tecidos comprimidos, assim como mudar a posição do corpo para aliviar a pressão. ${ }^{14}$

Salienta-se que os cuidados de enfermagem vão além das medidas citadas. Esses cuidados com as UPabrangem intervenções relacionadas ao acompanhamento integral do paciente em risco de adquirir a lesão por meio da utilização de escalas de predição de risco, conhecimento dos fatores de risco e da realidade das unidades de saúde. ${ }^{11}$

A Escala de Braden na prática clínica é um instrumento útil de predição para o desenvolvimento de UP ou sua recidiva, pois permite conhecer o risco individual de cada paciente e implementar precocemente ações de enfermagem preventivas e condizentes com este risco. ${ }^{17}$ Além da escala de Bradem, a mais citada na literatura, o uso das escalas de Norton e Waterlow também se mostra efetivo na predição de risco. ${ }^{18}$

Os cuidados relatados pelos enfermeiros não foram suficientes para evitar as úlceras por pressão. Isso remete à necessidade de se avaliar a qualidade dos cuidados e não apenas a descrição quantitativa, bem como a ampliação destes cuidados.

\section{CONSIDERAÇÕES FINAIS}

Mensurar qualidade e quantidade na assistência de enfermagem proporciona uma visão mais ampla dos fenômenos envolvidos, o que direciona para ações efetivas de acordo com as necessidades.

Ao analisarmos a incidência de UP, verificamos a baixa incidência na população geral, considerando todos os pacientes independente da presença de fatores de risco. Isso mostra que há bons indicativos de qualidade assistencial. Sob outra perspectiva, ao analisarmos os pacientes com úlcera, identificou-se que todos possuíam diversos fatores de risco, o que reforça a necessidade de avaliar os fatores de risco e atuar em prevenção.

Foram identificados que vários são os fatores de riscos que podem estar associados ao desencadeamento de UP e que diversos são os cuidados de enfermagem para prevenção. Apesar de se constatar a existência de ações de cuidado de enfermagem, houve o desenvolvimento de UP. Isso remete à necessidade de ampliar a coleta de informações quantitativas e qualitativas no processo de trabalho com vistas à identificação dos riscos, atuação frente a estes fatores e consequente diminuição dos casos de UP, bem como de sua gravidade.

Neste sentido, o trabalho de prevenção de UP passa diretamente por uma qualificada avaliação do risco, já que todos os pacientes que desenvolveram UP possuíam fatores de risco. Com a avaliação minuciosa, parte-se para as medidas de controle com métodos preventivos de acordo com a necessidade de cada paciente.

Considerando que cada instituição/cidade/estado/país possui realidades distintas que resultaram dos recursos envolvidos, políticas institucionais e diferenças culturais, considera-se necessária a identificação de indicadores assistências in locus para que as ações de melhoria sejam adequadas ao contexto.

Os diferentes índices de UP entre a literatura e este estudo mostram que é possível haver a subnotificação desse evento adverso. Este fato pode estar relacionado a rotatividade dos profissionais, bem como a problemas de comunicação e conhecimento das rotinas institucionalizadas. Identifica-se, portanto, a necessidade de ampliar-se o trabalho com vistas à avaliação da acurácia das notificações de eventos adversos. 


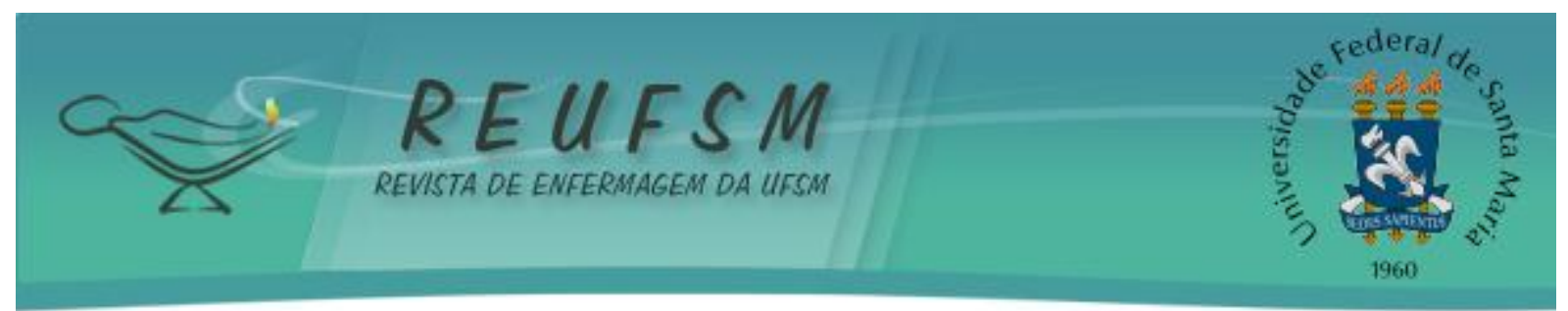

A avaliação dos processos de trabalho envolve a participação das diversas esferas envolvidas na atenção à saúde e uma mudança de atitude frente aos problemas identificados. É preciso superar ideias meramente punitivas e trabalhar com uma visão de construção humana e, consequentemente, de um trabalho de qualidade, na qual cada um desempenha um papel indispensável para o sucesso da assistência.

Este movimento passa pela Educação em Saúde, visando a formação profissional e humana por meio da educação em serviço e a busca pelo conhecimento cientifico para uma melhoria da qualidade da assistência prestada.

\section{REFERÊNCIAS}

1. Hess CT. Tratamento de feridas e úlceras. $4^{\mathrm{a}}$ ed. Rio de Janeiro: Reichmann \& Affonso; 2002. $226 \mathrm{p}$.

2. Dealey C. Cuidando de feridas: um guia para as enfermeiras. $3^{\text {a }}$ ed. São Paulo: Atheneu; 2008.

3. Bittar OJNV. Indicadores de qualidade e quantidade em saúde. Rev Adm Saúde. 2001 jul-set;3(12).

4. Polit DF, Beck CT, Hungler BP. Fundamentos de pesquisa em enfermagem: métodos, avaliação e utilização. Thorell A (trad.). $5^{\text {a }}$ ed. Porto Alegre: Artmed; 2004.

5. Richardson RJ. Pesquisa social: métodos e técnicas. $3^{a}$ ed. São Paulo: Atlas; 1999. 334 p.

6. Brasil. Conselho Nacional de Saúde. Diretrizes e normas regulamentadoras de pesquisa em seres humanos. Resolução N 196, de 10 de outubro de 1996. Brasília; 1996.

7. Diccini S, Camaduro C, Senyer LII. Incidência de úlcera por pressão em pacientes neurocirúrgicos de hospital universitário. Acta Paul Enferm [internet]. 2009 [acesso em 2011 set 23];22(2):205-9. Disponível em:

www.scielo.br/scielo.php?script=sci_arttext\&pid=S0103-21002009000200014\&lng=en.

8. Louro M, Ferreira M, Povoa P. Avaliação de protocolo de prevenção e tratamento de úlceras de pressão. Rev Bras Ter Intensiva. 2007;19(3):337-41.

9. Rocha JA, Miranda MJ, Andrade MJ. Abordagem terapêutica das úlceras de pressão: intervenções baseadas na evidência. Acta Med Port. 2006;19:29-38.

10. Prazeres SJ. Tratamento de feridas: teoria e prática. Porto Alegre: Muriá; 2009.

11. Medeiros ABF, Lopes CHAF; Jorge MSB. Análise da prevenção e tratamento das úlceras por pressão propostos por enfermeiros. Rev Esc Enferm USP. 2009;43(1).

12. Craven RF, Hirnle CJ. Fundamentos de enfermagem: saúde e funções humanas. $4^{\mathrm{a}}$ ed. Rio de Janeiro: Guanabara Koogan; 2006. 1584 p.

13. Irion GL. Feridas: novas abordagens, manejo clinico e atlas em cores. Rio de Janeiro: Guanabara Koogan; 2005.

14. Malagutti W, Kakiara CT. Curativo, estomias e dermatologia: uma abordagem multiprofissional. São Paulo: Martinari; 2010. 


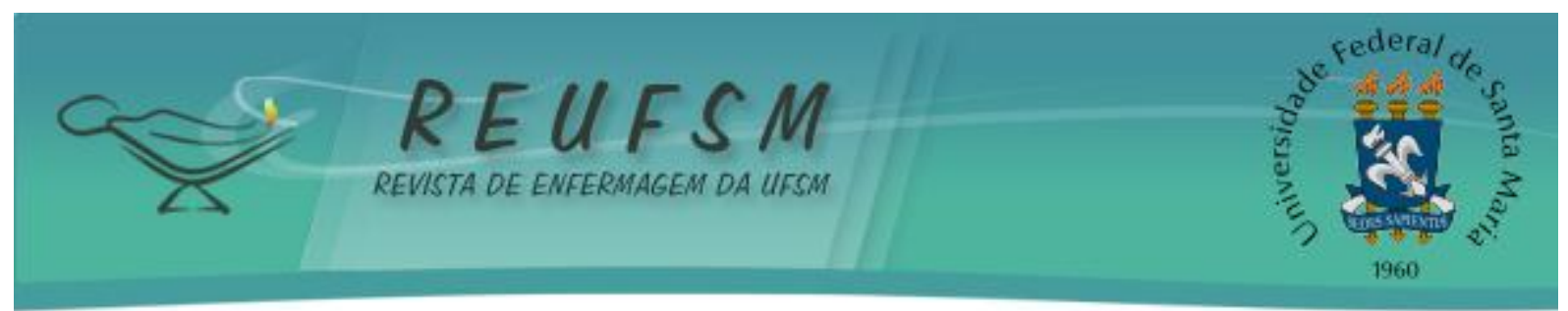

15. Nogueira PC, Caliri MHL, Santos CB. Fatores de risco e medidas preventivas para ulcera de pressão no lesado medular. Experiência da equipe de enfermagem do Hospital das Clínicas da FMRP-USP. Medicina (Ribeirão Preto). 2002 jan/mar;35:14-23.

16. Gomes FSL, Bastos MAR, Matozinhos FP, Temponi HR, Velásquez-Meléndez G. Fatores associados à úlcera por pressão em pacientes internados nos Centros de Terapia Intensiva de Adultos. Rev Esc Enferm USP. 2010;44(4):1070-6.

17. Gomes FSL, Bastos MAR, Matozinhos FP, Temponi HR, Velásquez-Meléndez G. Avaliação de risco para úlcera por pressão em pacientes críticos. Rev Esc Enferm USP. 2011;45(2):313-8.

18. Araújo, TM. Acurácia de escalas de avaliação de risco para úlcera por pressão em pacientes críticos [dissertação]. Fortaleza: Universidade Federal do Ceará; 2009. 149 f.

Data de recebimento: $13 / 04 / 2012$

Data de aceite: 20/06/2012

Contato com autor responsável: Mara Rosane Vargas e Silva Endereço: Rua Adão Pinheiro da Silva, 615. Bairro Aberta dos Morros.

CEP: 91751-030

E-mail: mara@brigadamilitar.rs.gov.br 